Boise State University

ScholarWorks

Computer Science Faculty Publications and Presentations

Department of Computer Science

4-2021

\title{
Somewhere Over the Rainbow: Exploring the Sense for Relevance in Children
}

\author{
Monica Landoni \\ Università della Svizzera Italiana \\ Theo Huibers \\ University of Twente \\ Emiliana Murgia \\ Università degli Studi di Milano-Biocca \\ Mohammad Aliannejadi \\ University of Amsterdam \\ Maria Soledad Pera \\ Boise State University
}

\section{Publication Information}

Landoni, Monica; Huibers, Theo; Murgia, Emiliana; Aliannejadi, Mohammad; and Pera, Maria Soledad. (2021). "Somewhere Over the Rainbow: Exploring the Sense for Relevance in Children". ECCE 2021: European Conference on Cognitive Ergonomics 2021, 30. https://doi.org/10.1145/3452853.3452885

This document was originally published in ECCE 2021: European Conference on Cognitive Ergonomics 2021 by the Association for Computing Machinery. Copyright restrictions may apply. https://doi.org/10.1145/3452853.3452885 


\section{Somewhere over the Rainbow: Exploring the Sense for Relevance in Children}

\author{
Monica Landoni \\ monica.landoni@usi.ch \\ Università della Svizzera Italiana \\ Lugano, Switzerland
}

\author{
Theo Huibers \\ University of Twente \\ Enschede, The Netherlands \\ t.w.c.huibers@utwente.nl
}

\author{
Emiliana Murgia \\ Università degli Studi di \\ Milano-Bicocca \\ Milano, Italy \\ emiliana.murgia@unimib.it
}

\author{
Mohammad Aliannejadi \\ University of Amsterdam \\ Amsterdam, The Netherlands \\ m.aliannejadi@uva.nl
}

\author{
Maria Soledad Pera \\ People and Information Research \\ Team (PIReT) - Boise State University \\ Boise, Idaho, USA \\ solepera@boisestate.edu
}

\begin{abstract}
We explore the facets of relevance that guide children when assessing materials retrieved by search engines when looking for information in the classroom. We involved children in a collaborative exercise and asked them to design innovative icons to point their peers towards useful results. We also asked them to complete a survey meant to capture explicit motivators guiding their design. This resulted in a rich set of metaphors. Analysis of the emerging metaphors is what allowed us to identify and discuss the many interpretations of relevance children naturally assign to resources they find in response to school-related information discovery tasks.
\end{abstract}

\section{CCS CONCEPTS}

- Social and professional topics $\rightarrow$ Children; • Information systems $\rightarrow$ Web search engines; Relevance assessment.

\section{KEYWORDS}

children, search, SERP, classroom, relevance, emoji, interpretation, GUI, user study

\section{ACM Reference Format:}

Monica Landoni, Theo Huibers, Emiliana Murgia, Mohammad Aliannejadi, and Maria Soledad Pera. 2021. Somewhere over the Rainbow: Exploring the Sense for Relevance in Children. In European Conference on Cognitive Ergonomics 2021 (ECCE 2021), April 26-29, 2021, Siena, Italy. ACM, New York, NY, USA, 5 pages. https://doi.org/10.1145/3452853.3452885

\section{INTRODUCTION}

Children turn to search engines (SE) for completing informationseeking tasks related to school assignments. Unfortunately, mainstream SE are not designed to meet the needs of young searchers.

Permission to make digital or hard copies of all or part of this work for personal or classroom use is granted without fee provided that copies are not made or distributed for profit or commercial advantage and that copies bear this notice and the full citation on the first page. Copyrights for components of this work owned by others than the author(s) must be honored. Abstracting with credit is permitted. To copy otherwise, or republish, to post on servers or to redistribute to lists, requires prior specific permission and/or a fee. Request permissions from permissions@acm.org.

ECCE 2021, April 26-29, 2021, Siena, Italy

(C) 2021 Copyright held by the owner/author(s). Publication rights licensed to ACM. ACM ISBN 978-1-4503-8757-6/21/04 ..\$15.00

https://doi.org/10.1145/3452853.3452885
Among the many struggles children face when interacting with SE $[8,11]$, we are particularly interested in children's perception of relevance, an imperative ingredient for success full completion of the search process.

A conventional view of relevance. Adult searchers simply scan through the snippets generated by SE in response to their inquiries to identify relevant resources. In most cases, users gauge resources' source and content to determine which ones match their information needs swiftly. An information need is "intangible and visceral and thus unknowable and non-specifiable in a query to an information system" [6]; thus, it is not unexpected that many factors (e.g., education and digital literacy exposure) affect the notion of relevance concerning the information needs of an adult user. Literature describing models related to facets, dimensions, degrees, and the dynamic nature of relevance is rich $[5,7,14]$. Specific emphasis has been paid to the situational type of relevance (aka manifestation) [19]. In this case, relevance is determined by the usefulness of the retrieved information for concluding a given task. In the end, adult searchers decide which resources retrieved by SE are good, i.e., relevant, on their own volition.

Children's perspectives on SE use. If we shift the focus to young searchers, recent analyses of SE result pages (SERP) generated by mainstream SE in response to children's queries reveal a complexity on the content of presented snippets [4]. This is a concern, as if these young users cannot comprehend the content of the snippets, it will hardly be possible for them to decide on the corresponding degree of relevance. Further, resources prioritized by SE in response to children's inquiries are not necessarily tailor-made for them [2, 4]. With children following a linear exploration pattern, deeming resources relevant most often than not because they appear early in the ranking of retrieved resources [11], it is imperative to adapt SERP for them so that they can support children resource discovery more effectively.

Children's view of relevance. Enriching SERP to guide children requires that we first understand what children view as relevant and how to best capture this concept. Doing so involves exploring the different types and many facets of relevance that guide children when assessing resources retrieved by SE. We argue that situational 
relevance and in particular the motivational/affective "inherent characteristics of relevance behaviour" [5], have to be considered when studying children searching in the classroom. In this case, the former focuses on the usefulness of retrieved materials concerning tasks assigned by teachers; whereas the latter accounts for the goals and motivations behind searching for learning. While usefulness is naturally assessed by teachers, the motivational/affective type of relevance is more complex to study when young searchers are involved, making it an interesting area for us to further investigate.

Children's perception of motivational relevance: metaphors. We begin our exploration by conducting a user study where we treated children as experts. We involved children in a collaborative exercise and asked them to design innovative SERP to point their peers to useful SE results. We presented children with alternative icons to foster their creativity and willingness to share their opinions. Thereafter, we examined children's views, captured via responses to a survey and drawings. This resulted in a rich set of metaphors that sum up what children see as clues for relevance for the classroom. Through metaphor analysis, we explore the many interpretations children naturally assign to the material they find relevant to a given task. In the rest of this manuscript, we describe the setup of our study and discuss preliminary findings. To control the scope of our study, we follow the framework defined in [11], which establishes four pillars-user group, task, context and strategy-to evaluate information retrieval systems for children: (i) children in primary five; (ii) searching for resources in the classroom; (iii) in response to curriculum-related inquiries; (iv) using alternative graphical user interfaces (GUI) for search, each including icons portraying different metaphors.

\section{STUDY SET-UP}

We describe below the user study we conducted to gather data to enable the analysis of children's view of relevance.

Participants. We involved nine children (ages 10-11, five boys and four girls) in a collaborative exercise where we considered them experts. To align with the study goal, we sought children who have regular exposure and instruction related to search tools and participated in previous studies involving co-designing interfaces for information retrieval systems tailored to children and the classroom $[11,18]$. Children attend primary five at schools that follow the same curriculum; thus they have comparable reading skills and share a similar concept as to what constitutes a useful result. Recruitment was voluntary. We obtained parental consent in advance; the local Ethics committee approved the study.

Protocol. The prompt initiating the study was for children to design innovative SERP to point their peers to useful resources that could foster the completion of classroom-related inquiry tasks. We conducted our study in 3 stages. ${ }^{1}$ In the first stage, and to motivate discussion, we presented children with three options of icons to depict relevance: a popular one (Figure 1a), a fun one (Figure 1b), and an image children are familiar with (Figure 1c), with a red light for content not worth opening and a green one for useful content) In the second stage, we asked children to share their opinions on

${ }^{1}$ Due to page-limitations, we omit details; instead, please turn to [1], as we replicate the protocol originally proposed by the authors. the icons as mentioned above and their idea of relevance (via a survey). In the third stage, we invited children to be creative and sketch new icons to enhance SERP and describe succinctly why they would design them that way. Using metaphors as the driver for our exploration, we then gathered children's views. Metaphors are rhetorical figures used to introduce new concepts by referring to well-known ones, sharing some or most of the look and feel as well as functionalities. A working metaphor usually arises from a familiar look that implies a straightforward modality of interaction. Icons on GUI are common examples of easy-to-recognise metaphors, e.g., folders to store documents. Indeed, metaphors based on familiar icons help users make sense of how to confidently interact with new applications. Each metaphor is defined in terms of its signifier and related affordance. A signifier is a visual indicator carrying critical information for the user to trigger references to familiar concepts [16]. Affordance is the action suggested to a user when interacting with an artifact [15], in our case, the proposed icon.

Virtual interaction. This study took place during the COVID pandemic. As such, it was conducted remotely-guided by an educator via Skype and taking advantage of collaborative tools, e.g., Google Forms and Google Docs.

\section{PRELIMINARY RESULTS AND ANALYSIS}

We explore what metaphors children naturally refer to when describing results they find useful. To stimulate their creativity, we present children with alternative ways to represent the binary dichotomy between useful and useless (Figure 1). We also gather child experts' feedback (using prompts including "Which icon would you use if you were looking for information on tornados? Why?" and user experience questionnaires including Likert scales for some icon trains, as listed in Figure 2) and engage them in sketching their proposed alternatives. We proceed to examine their suggested designs, from which we infer a rich set of metaphors that help us better understand their view of what "useful" really stands forwhat do children see as clues for relevance for the classroom? As in [10], we focus our analysis on the concept of signifier as depicted in children's drawings and link it to its related affordance as described by children in the survey so to complete the underlining metaphors.

We discuss below children's feedback on the presented alternative GUI enriched with different icons.

- Thumbs up-down - Approved or not: The signifiers were clear, representing something approved or not; children intuitively perceived the affordance of these icons and interpreted them as expressive of the degree to which a result was worth clicking on. Children judged this icon as the easiest, most supportive, and clear (Figure 2). Nonetheless, they mentioned that when scrolling fast across SERP, the thumbs up and down could get confused, i.e., not be uniquely recognisable on the move.

- Rainbow and Poo - Pleasant or smelly: These signifiers were the most fun and cheerful; children elected this pair as the one their classmates would prefer. Across all participants, these icons were the most exciting and interesting, faring very well also as inventive and leading-edge (Figure 2). The affordance was clear, too, even if the poo icon had a 


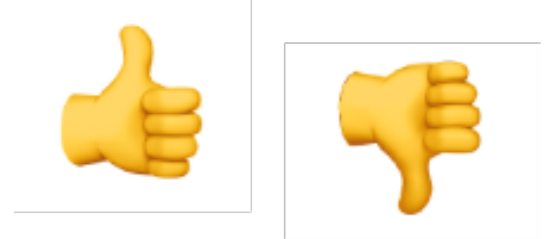

(a) Socially Popular

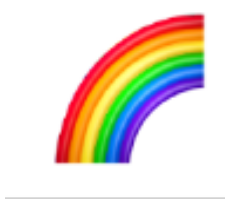

(b) Fun

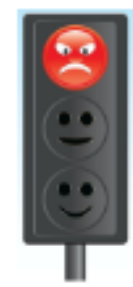

(c) Classic

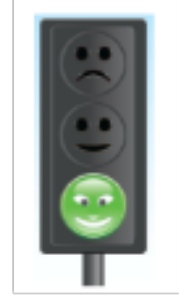

Figure 1: Icons presented to children to prompt their discussion on the different facets of relevance.

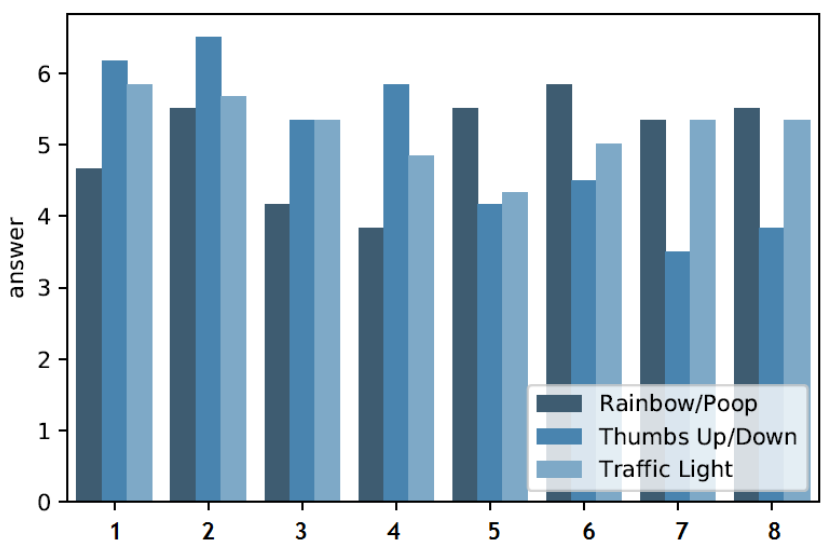

Figure 2: Children's opinions on icons in Fig. 1. X-axis includes some pre-defined characteristics to prompt feedback gathering (1) Obstructive vs. Supportive; (2) Complicated vs. Easy; (3) Inefficient vs. Efficient; (4) Confusing vs. Clear; (5) Boring vs. Exciting; (6) Not Interesting vs. Interesting; (7) Conventional vs. Inventive; (8) Usual vs. Leading Edge. Score ranged from 0 (lowest, e.g., "Obstructive”) to 7 (highest, e.g., "Supportive"). Y-axis: Average score assigned by children.

cheerful smile that could be misconstrued as an invitation to click on it. Still, children's interpretation was straightforward: rainbow-tagged results were pleasant and thus worth clicking on, whereas those tagged with poo were "smelly" (i.e., unpleasant) and therefore should be avoided. However, when taking context into account, children agreed that this fun option was not suitable for school.

- Green and Red light - Go or stop: Both signifiers and affordance were very clear. Children agreed red signified stop and green go ahead, implying an invitation to click and explore. As showcased in Figure 2, Green/red light icons challenged the Rainbow-poo counterpart, as being inventive and leading-edge; this combination also offered a similar level of support as the Thumb-up/down. When asked about whether their classmates would use and like it, children observed that relying on red and green colours could be difficult for color-blind individuals. This concern shines a light on the need for icons to be more inclusive and accessible to all.

Important outcomes from our collaborative exercise emerged from children drawing their own icons (a sample shown in Figure 3). Literature reports how drawing sessions are used to elicit user preferences when designing for and with children $[13,17]$ as an effective means to capture and communicate complex concepts. Here we use the analysis of drawings made by children to understand their interpretation of relevance. The suggested icons (i) offer insights into the qualities expected of resources in SERP to be clicked and (ii) inform the interpretation of the many facets of useful in the context of searches conducted in the classroom environment.

- Angel and Evil - Good or bad: These signifiers portrait the most classic dichotomy of all, an interpretation of good (i.e., useful) and bad (i.e., useless) that opens a discussion on the kind of content children are afraid to find online and their need to feel protected. The implied affordance would be an open invitation to trust and safely open the pages marked with an angel and keep well away from those with the devil.

- Switched on and off bulb - Light or darkness: Here, the signifier describes a tension between content that can enlighten searchers by providing useful insights and its opposite, which actually causes confusion. One child added the term reliable and non-reliable next to the icons explicitly, as reliable content is a way to switch on the light and see further, and non-reliable one muddles things up by hiding information and making it non-visible. In terms of affordance, children would click on the lighted bulb if looking for clarifications and avoid results marked with the switched-off bulb to stay away from confusion.

- Open/closed window - Explore or avoid: An open window is a signifier that brings an invitation, together with an affordance to explore further. A closed one is precluding any further browsing. By blocking access to a result, children prevent their peers from wasting time without expressing a judgment on the retrieved information's quality.

- Ticked and Crossed - Right or wrong: By using these signifiers, as teachers do when marking school assignments, children conveyed the right and wrong metaphor. The associated affordance is an invitation to their peers to look only into the right content, a choice in line with the school context. The tick conveys a sense of being approved and deemed suitable for them to be read. 


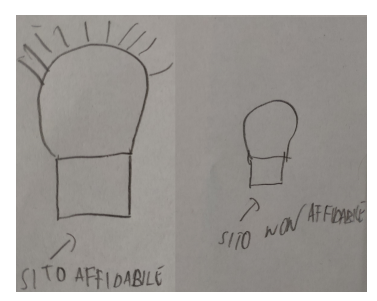

(a) Lightbulbs (reliable, not reliable on original Italian)

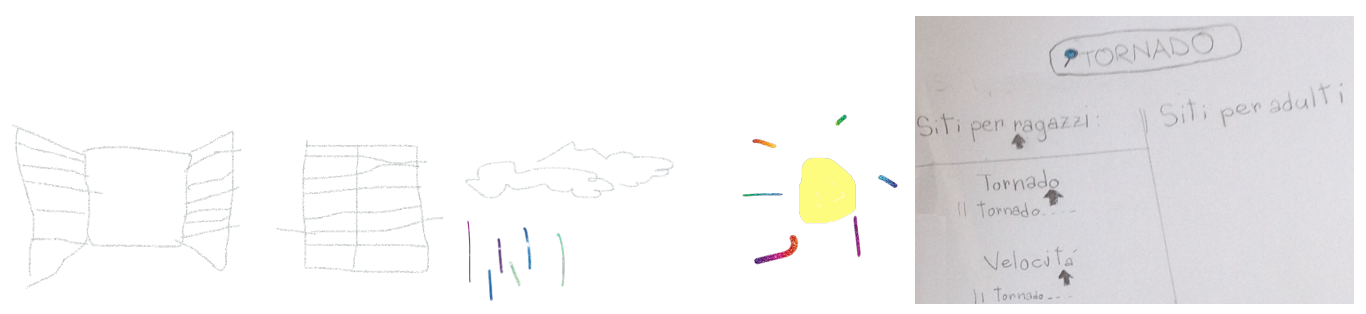

(b) Windows (c) Weather (d) Single Arrow

Figure 3: Exploring metaphors: icons created by children to tag resources useful for completing classroom tasks.

- Arrow up - For children or for adults: Arrows pointing up are used to tag useful results, i.e., suitable for children, and keep them visually separated from those for more mature users. This is the only design proposing a single icon not paired with its opposite.

- Smile and sad - Positive or negative: With a smile and a sad face, children hinted at positive results being perceived as more clickable than those ranked highly by the SE, in line with the literature $[9,12]$.

- Sun and Rain - Desirable or to be avoided: The implication here is that the sun is more desirable than the rain. In this case, associating results to something more desirable would make it more clickable, in turn actively disregarding results tagged with rain.

Discussion and limitations. We neither discussed nor engaged children in any activity exploring the different shades of relevancewe simply classify results following the scoring method introduced by Bilal [3]: relevant or not to be missed, irrelevant or to be avoided, and semi-relevant or neutral. Further, we used familiar binary icons expressing opposite concepts. We studied the nuances of their meaning and their implicit bias when placed next to results in SERP, in terms of prompting children to click them when conducting school-related searches. In summary, children interpret relevance as good and suitable for them, enlightening and reliable, inviting and conducive to more discoveries, and the more traditional right and correct. These interpretations help us shed light on the concept of affective/motivational relevance and how young searchers make sense of it to feel motivated to search further and trust the SE.

\section{CONCLUSIONS AND FUTURE WORK}

We explored how children perceive and interpret relevance when searching for resources related to school-related inquiry tasks. By engaging children as experts and asking them to assess, propose, sketch, and discuss metaphors representing dichotomies related to relevance, we grasped a preliminary sense of what makes a result clickable for them. More work is needed in order to model the many nuances and shades of relevance for young searchers, starting with broader age-ranges (as age can be a factor affecting the perception of children's relevance) and considering the concept of personalised SERP to explore the influence of children's chosen metaphors on their resource relevance judgments. The next steps also involve offering icon-enhanced SERP to children and engage them with searches in the classroom to gather quantitative and qualitative data about their interactions with search results. This will allow us to expand our initial set of possible interpretations of what a relevant result needs to be for children to click on it, beyond being useful. We also plan to capture how result quality (e.g., inviting and conducive to discovery) influences the information-seeking process across age, developmental level of children, and their familiarity with technology. This will open new research paths towards the design of innovative search tools to support children when searching in the classroom.

\section{ACKNOWLEDGMENTS}

We would like to thank the children in Switzerland and Italy, for their willingness to engage in our study online.

\section{REFERENCES}

[1] Mohammad Aliannejadi, Theo Huibers, Monica Landoni, Emiliana Murgia, and Maria Soledad Pera. 2020. Say it with Emojis: Co-designing Relevance Cues for Searching in the Classroom. In CIRCLE. http://ceur-ws.org/Vol-2621/CIRCLE20_ 30.pdf

[2] Oghenemaro Anuyah, Ashlee Milton, Michael Green, and Maria Soledad Pera. 2019. An empirical analysis of search engines' response to web search queries associated with the classroom setting. Aslib (2019).

[3] Dania Bilal. 2000. Children's use of the Yahooligans! Web search engine: I. Cognitive, physical, and affective behaviors on fact-based search tasks. FASIST 51, 7 (2000), 646-665.

[4] Dania Bilal and Li-Min Huang. 2019. Readability and word complexity of SERPs snippets and web pages on children's search queries. Aslib (2019).

[5] Pia Borlund. 2003. The concept of relevance in IR. JASIST 54, 10 (2003), 913-925.

[6] Charles Cole. 2011. A theory of information need for information retrieval that connects information to knowledge. JASIST 62, 7 (2011), 1216-1231.

[7] Erica Cosijn and Peter Ingwersen. 2000. Dimensions of relevance. IP \& M 36, 4 (2000), 533-550.

[8] Jerry Alan Fails, Maria Soledad Pera, Oghenemaro Anuyah, Casey Kennington, Katherine Landau Wright, and William Bigirimana. 2019. Query formulation assistance for kids: What is available, when to help \& what kids want. In $A C M$ IDC. $109-120$.

[9] Gabriella Kazai, Paul Thomas, and Nick Craswell. 2019. The Emotion Profile of Web Search. In ACM SIGIR. 1097-1100.

[10] Jingoog Kim and Mary Lou Maher. 2019. Metaphors, Signifiers, Affordances, and Modalities for Designing Mobile and Embodied Interactive Systems. In ACM OZCHI. 542-545.

[11] Monica Landoni, Davide Matteri, Emiliana Murgia, Theo Huibers, and Maria Soledad Pera. 2019. Sonny, Cerca! evaluating the impact of using a vocal assistant to search at school. In CLEF. 101-113.

[12] Monica Landoni, Maria Soledad Pera, Emiliana Murgia, and Theo Huibers. 2020. Inside Out: Exploring the Emotional Side of Search Engines in the Classroom. In ACM UMAP. 136-144.

[13] Sunok Lee, Sungbae Kim, and Sangsu Lee. 2019. " What does your Agent look like?" A Drawing Study to Understand Users' Perceived Persona of Conversational Agent. In Extended Abstracts of ACM CHI. 1-6.

[14] Stefano Mizzaro. 1998. How many relevances in information retrieval? Interacting with computers 10, 3 (1998), 303-320. 
[15] Donald A Norman. 1988. The psychology of everyday things. Basic books.

[16] Donald A Norman. 2008. The way I see IT signifiers, not affordances. Interactions 15,6 (2008), 18-19.

[17] Mohammad Obaid, Wolmet Barendregt, Patricia Alves-Oliveira, Ana Paiva, and Morten Fjeld. 2015. Designing robotic teaching assistants: interaction design students' and children's views. In ICSR. Springer, 502-511.
[18] Maria Soledad Pera, Emiliana Murgia, Monica Landoni, and Theo Huibers. 2019. With a Little Help from My Friends: Use of Recommendations at School. In ACM RecSys 2019 Late-Breaking Results: Co-Located with ACM RecSys. 61-65.

[19] Tefko Saracevic. 1996. Relevance reconsidered. In CoLIS 2. ACM New York, 201-218. 